\title{
Cooperative Jahn-Teller Coupling in the Manganites
}

\author{
Z. Popovic and S. Satpathy \\ Department of Physics \& Astronomy, University of Missouri, Columbia, Missouri 65211
}

(Received 8 June 1999)

\begin{abstract}
The cooperative Jahn-Teller coupling between the Mn $e_{g}$ electrons and the oxygen octahedral distortions in $\mathrm{LaMnO}_{3}$ is studied using ab initio density-functional calculations and tight-binding models. The linear and quadratic vibronic coupling parameters are calculated using density-functional methods. It is shown that the cooperative Jahn-Teller coupling, primarily due to the interoctahedral electron hopping (band structure term), leads to the ordering of the octahedral distortion and simultaneously to orbital ordering. The coupling results in a two-minima adiabatic potential surface in the solid, instead of the three-minima "Mexican-hat" surface for the isolated octahedron.
\end{abstract}

PACS numbers: 75.30.Vn, 31.30.Gs

An important piece of physics in the colossal magnetoresistive manganites is the Jahn-Teller (JT) coupling between the $e_{g}$ electrons and the distortion modes of the $\mathrm{MnO}_{6}$ octahedron. This coupling plays an integral role in transport [1] and is manifested beautifully in the isotope effect [2,3]. This so-called $E \times e$ vibronic coupling has been studied quite well for the isolated JT center [4], and these results have been widely used to interpret the vibronic coupling in the manganites. We show here that the vibronic coupling for the isolated center is modified by interaction between the individual centers in the crystal (cooperative Jahn-Teller effect) mediated via electron hopping. Focusing on $\mathrm{LaMnO}_{3}$, we show that this coupling results in the simultaneous ordering of the octahedral distortions as well as of the $e_{g}$ orbitals as observed in the $\mathrm{LaMnO}_{3}$ crystal.

Consider first the isolated $\mathrm{MnO}_{6}$ octahedron. The vibronic JT coupling in this case is described by the wellknown Hamiltonian

$$
\mathcal{H}=\frac{1}{2} K Q^{2}+\mathcal{H}_{\mathrm{JT}},
$$

where $Q^{2}=Q_{1}^{2}+Q_{2}^{2}+Q_{3}^{2}$, with the $Q$ 's being the three active octahedral distortion modes. The JT Hamiltonian is given by [4-6]

$$
\begin{aligned}
\mathcal{H}_{\mathrm{JT}}= & -g\left(Q_{2} \tau_{x}+Q_{3} \tau_{z}\right) \\
& -G\left(Q_{3}^{2}-Q_{2}^{2}\right) \tau_{z}+2 G Q_{2} Q_{3} \tau_{x},
\end{aligned}
$$

where both the linear and the quadratic vibronic coupling terms have been included, the respective coefficients being $g$ and $G$, and $\vec{\tau}$ is the pseudospin describing the two $e_{g}$ orbitals, viz., $|\uparrow\rangle=\left|z^{2}-1\right\rangle$ and $|\downarrow\rangle=\left|x^{2}-y^{2}\right\rangle$. For the case of the isolated octahedron, this leads to the well-known "Mexican-hat" type adiabatic potential surface with three energy minima in the $Q_{2}-Q_{3}$ plane along $\phi=0, \pm 2 \pi / 3$, where $\phi \equiv \tan ^{-1}\left(Q_{2} / Q_{3}\right)$ [4].

In the solid, the above expression for the Hamiltonian is modified because first, the vibronic coupling strength for different modes could be different due to the reduction of the octahedral symmetry in the crystal, and second, and more importantly, two additional terms, viz., the band structure term and the elastic energy term between neighboring octahedra, should now be included. The Hamiltonian in the crystal may be written in the following form [7]:

$$
\begin{aligned}
\mathcal{H}_{\text {cryst }}= & \sum_{i} \frac{1}{2} K Q_{i}^{2}+K^{\prime} \sum_{\langle i j\rangle_{\alpha}} \tilde{Q}_{i \alpha} \tilde{Q}_{j \alpha} \\
& +\sum_{i} \mathcal{H}_{\mathrm{JT}}^{i}+\mathcal{H}_{\mathrm{ke}},
\end{aligned}
$$

where in the elastic interaction term between neighboring octahedra, the $\tilde{Q}_{\alpha}$ 's are defined as $\tilde{Q}_{x}=\left(Q_{3}+\right.$ $\left.\sqrt{3} Q_{2}\right) / 2, \tilde{Q}_{y}=\left(Q_{3}-\sqrt{3} Q_{2}\right) / 2, \tilde{Q}_{z}=Q_{3}$, and $\langle i j\rangle_{\alpha}$ are the nearest-neighbor (NN) pairs of octahedra along the direction $\alpha=\hat{x}, \hat{y}$, or $\hat{z}$. The last two terms in Eq. (3) are the electronic structure terms for the $e_{g}$ electrons: $\mathcal{H}_{\mathrm{JT}}^{i}$ is the intra-octahedral JT term [Eq. (2)] at the $i$ th Mn site and $\mathcal{H}_{\mathrm{ke}}$ is the kinetic energy due to the inter-octahedral hopping of the $e_{g}$ electrons in the lattice. Adopting a tight-binding model for the latter, we write

$$
\mathcal{H}_{\mathrm{ke}}=\sum_{\langle i j\rangle, \sigma} \sum_{a b} V_{i j}^{a b} c_{i a \sigma}^{\dagger} c_{j b \sigma}+\text { H.c. }-J_{H} \sum_{i, a} S_{i} \cdot \sigma_{i a},
$$

where $\sigma$ is the electron spin, $i$ is the site index, and $a$ is the orbital index. The $t_{2 g}$ core spins $S$ are considered classical and localized, and, furthermore, we take the Hund's-rule energy $J_{H}$ to be $\infty$ as appropriate for the manganites. The hopping integral is $V$, which depends on the relative positions of the two $\mathrm{Mn}$ sites. For $\mathrm{NN}$ along $\hat{x}$, e.g., we have [8]

$$
V^{a b}=\left(\begin{array}{cc}
1 & -\sqrt{3} \\
-\sqrt{3} & 3
\end{array}\right) \times \frac{V_{d d \sigma}}{4} .
$$

With the above model, we now obtain the band structure of the $e_{g}$ electrons in $\mathrm{LaMnO}_{3}$ in the "type $A$ " antiferromagnetic structure. Since the magnetization of the successive ferromagnetic $a b$ planes alternate along the $c$ direction, we need consider only planar hopping along the $\mathrm{Mn}-\mathrm{O}$ plane. This is because the interplanar hopping is suppressed due to the Anderson-Hasegawa $\cos (\theta / 2)$ factor $[9,10]$ in the limit $J_{H} \rightarrow \infty$.

Keeping two Mn sites in the planar unit cell, so as to allow the possibility of the staggered distortion seen in 
$\mathrm{LaMnO}_{3}$, the tight-binding $e_{g}$ bands are obtained by the diagonalization of a $4 \times 4$ Hamiltonian:

$$
H(\vec{k})=\left(\begin{array}{cc}
A\left(Q_{2}, Q_{3}\right) & C \\
C & A\left(Q_{2}^{\prime}, Q_{3}^{\prime}\right)
\end{array}\right)
$$

where $\left(Q_{2}, Q_{3}\right)$ and $\left(Q_{2}^{\prime}, Q_{3}^{\prime}\right)$ are the distortions of the two Mn JT centers, $\vec{k}$ is the Bloch momentum, and $A$ and $C$ are the $2 \times 2$ matrices:

$$
A\left(Q_{2}, Q_{3}\right)=\left(\begin{array}{cc}
-g Q_{3}-G\left(Q_{3}^{2}-Q_{2}^{2}\right)+\alpha V_{\sigma}^{\prime} & -g Q_{2}+2 G Q_{2} Q_{3} \\
-g Q_{2}+2 G Q_{2} Q_{3} & g Q_{3}+G\left(Q_{3}^{2}-Q_{2}^{2}\right)+4 \alpha V_{\pi}^{\prime}
\end{array}\right)
$$

and

$$
C=\left(\begin{array}{cc}
\alpha_{+} V_{\sigma} & -\sqrt{3} \alpha_{-} V_{\sigma} \\
-\sqrt{3} \alpha_{-} V_{\sigma} & 3 \alpha_{+} V_{\sigma}
\end{array}\right)
$$

In the expressions above, only the first- and the secondneighbor hopping terms have been kept with $\alpha_{ \pm}(\vec{k})=\left(\cos k_{x} a \pm \cos k_{y} a\right) / 2, \alpha=\cos k_{x} a \times \cos k_{y} a$, and $\left(V_{\sigma}, V_{\pi}\right)$ and $\left(V_{\sigma}^{\prime}, V_{\pi}^{\prime}\right)$ are the Mn $d-d$ hopping integrals between the first and the second neighbors, respectively.

The Hamiltonian becomes especially simple at the $R$ point in the Brillouin zone, $\vec{k}=(1,1,1) \times(\pi / 2 a)$, yielding eigenvalues identical to those for the isolated octahedron,

$$
\epsilon^{ \pm}(R)= \pm Q\left[g^{2}+G^{2} Q^{2}+2 G g Q \cos 3 \phi\right]^{1 / 2},
$$

with $Q \equiv \sqrt{Q_{2}^{2}+Q_{3}^{2}}$ and $\phi \equiv \tan ^{-1}\left(Q_{2} / Q_{3}\right)$. To the second order in $Q$, this yields

$$
\Delta \epsilon \equiv \epsilon^{+}(R)-\epsilon^{-}(R)= \begin{cases}2 g Q_{2} & \left(Q_{2} \text { only }\right) \\ 2 Q_{3}\left(g+G Q_{3}\right) & \left(Q_{3} \text { only }\right) .\end{cases}
$$

Thus, the two vibronic coupling constants, $g$ and $G$, may be obtained from the splitting of the two doubly degenerate $e_{g}$ bands at the $R$ point, by considering pure $Q_{2}$ and $Q_{3}$ distortions.

To this end, we have performed a series of densityfunctional band calculations for $\mathrm{LaMnO}_{3}$ in the localdensity approximation (LDA) with the self-consistent linear muffin-tin orbitals (LMTO) method in the atomic spheres approximation (ASA), with controlled amounts of $Q_{2}$ or $Q_{3}$. (Density-functional bands for the observed crystal structure have been discussed in Refs. $[11,12]$.) Figure 1 shows the LDA bands for three different values of $Q_{2}$. From these calculations, we have extracted the gap value at the $R$ points, which are plotted in Fig. 2 as a function of the distortion strength. By fitting the gap value to Eq. (9), we find the coefficients $g=2.05 \mathrm{eV} / \AA\left(Q_{2}\right.$ mode) and $g=1.6 \mathrm{eV} / \AA$ and $G=2 \mathrm{eV} / \AA^{2}$ ( $Q_{3}$ mode). The slightly different values of $g$ for the two modes is consistent with the fact that the octahedral symmetry of the $\mathrm{MnO}_{6}$ unit is reduced in the crystal. For simplicity, we shall take $g=2.0 \mathrm{eV} / \AA$ for both the $Q_{2}$ and the $Q_{3}$ modes in the rest of the paper.

We have also fitted the $e_{g}$ bands in the LMTO band structure with the tight-binding bands obtained from Eq. (6), which yields the following values for the parameters: $g=2.0 \mathrm{eV} / \AA, G=1.5 \mathrm{eV} / \AA^{2}, V_{\sigma}=-0.52 \mathrm{eV}$,
$V_{\sigma}^{\prime}=-0.42 \mathrm{eV}, V_{\pi}^{\prime}=0.09 \mathrm{eV}$, and $V_{\sigma}^{\prime \prime}=-0.06 \mathrm{eV}$. A small third-neighbor hopping $V_{\sigma}^{\prime \prime}$ was necessary to obtain the proper shapes for the $e_{g}$ bands. The quality of the fit, as indicated from Fig. 1(c), is remarkably good given the simplicity of our tight-binding model. The magnitude of the second-neighbor hopping $V_{\sigma}^{\prime}$ is larger than one might expect from the Harrison scaling [8], but is not surprising considering that the hopping integrals originate from the overlap between the appropriate Wannier functions. Interestingly enough, a significant strength for the second-neighbor Mn-Mn hopping has been invoked by Jaime et al. to explain the Hall data [14].

Following earlier authors [15], we take the magnitude of the stiffness constant $K \approx 10 \mathrm{eV} / \AA^{2}$ from the optical data for $\mathrm{La}_{1.85} \mathrm{Sr}_{0.15} \mathrm{MnO}_{3}$, identifying the highest-lying phonon frequency $\omega_{\mathrm{ph}} \sim 70 \mathrm{meV}$ with the $\mathrm{Mn}-\mathrm{O}$ bondstretching mode [16]. Now, one could in principle compute $K$ from LDA by stretching a single $\mathrm{Mn}-\mathrm{O}$ bond in a supercell calculation. However, this being too cumbersome, we have contented ourselves by computing the LDA total energy for a uniform volume change using the full-potential and the ASA-LMTO methods [17]. The extracted value of $K_{\text {eff }}=20 \pm 2 \mathrm{eV} / \AA^{2}$ is, however, only an upper bound to $K$, since there are other "spring constants"
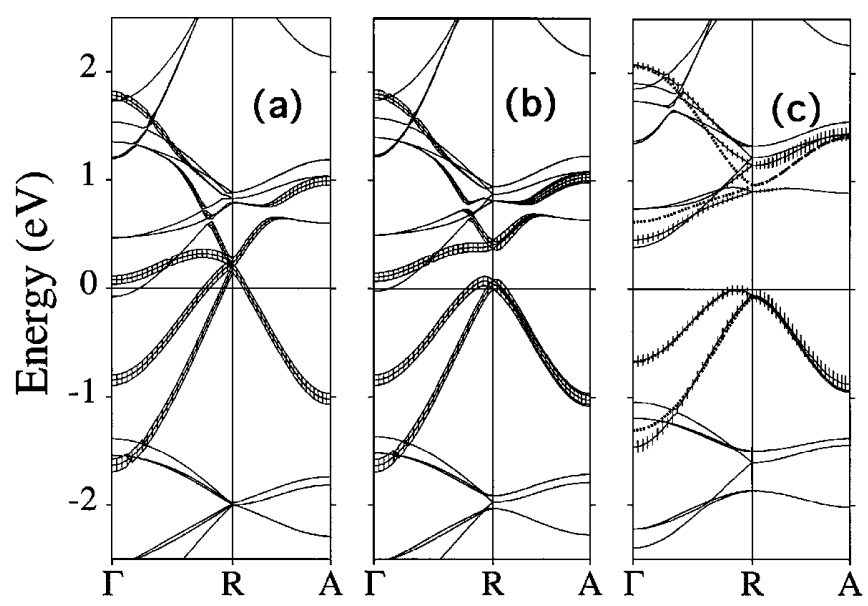

FIG. 1. LDA-LMTO bands for $\mathrm{LaMnO}_{3}$ in the "type- $A$ " structure with varying $Q_{2}$ distortions added to the ideal cubic crystal: $Q_{2}=0$ (a), $0.086 \AA$ (b), and $0.259 \AA$ (c). A volume preserving lattice constant $a=3.94 \AA$ was used. The $\vec{k}$ points are $\Gamma=(0,0,0), R=(1,1,1)$, and $A=(1,0,1)$ in units of $\pi /(2 a)$ with cube axes along Mn-O bonds. The LDA bands are given a width proportional to the contribution of the $e_{g}$ orbitals ("fat bands") [13]. Dotted lines in (c) show the tight-binding fit to the $e_{g}$ bands which are crosshatched. 


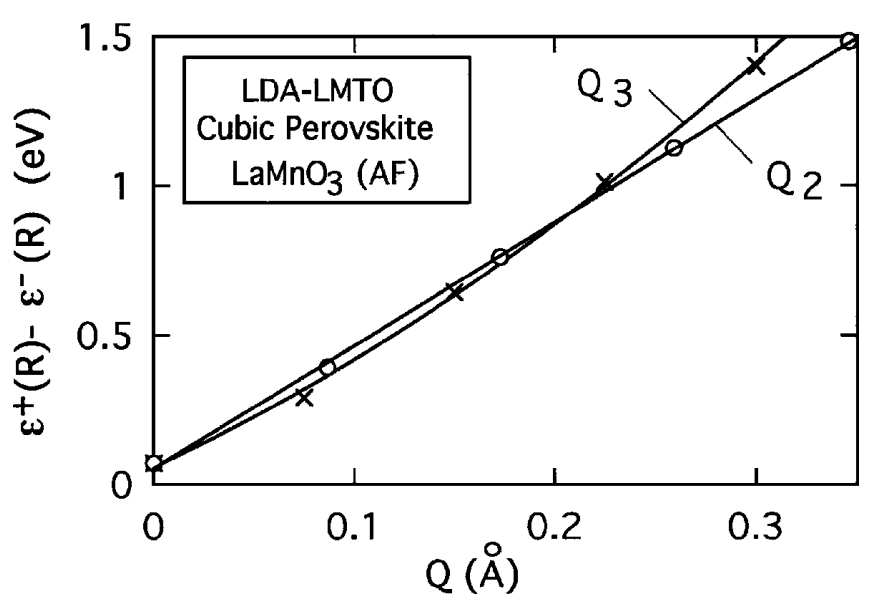

FIG. 2. Variation of the band gap at the $R$ point calculated from the LDA with the magnitude of the octahedral distortion. The variation follows Eq. (9).

involved in the total energy in addition to the stretching of the $\mathrm{Mn}-\mathrm{O}$ bond.

Turning now to the effect of the band structure term on the adiabatic potential surface, we compute the band structure energy from the occupied eigenvalues of (6), $E_{\text {band }}=\sum_{\nu \vec{k}}^{\epsilon_{F}} \epsilon(\nu, \vec{k}), \epsilon_{F}$ being the Fermi energy and $\nu$ the band index, as a function of the $\left(Q_{2}, Q_{3}\right)$ distortions. We considered all possible distortions of the type $\left( \pm Q_{2}, \pm Q_{3} ; \pm Q_{2}, \pm Q_{3}\right)$, where the first (last) two quantities correspond to the distortion of the first (second) JT center in the unit cell. Note that the observed crystal structure [18] has the distortion of the type $\left(Q_{2}, Q_{3} ;-Q_{2}, Q_{3}\right)$, with $Q_{2} \approx 0.28 \AA$ and $Q_{3} \approx-0.10 \AA$, with the convention of Ref. [19] for the normal modes.

To identify the energy gained due to the band formation, we define the band energy $\Delta E$ as

$$
\Delta E=\sum_{i} E_{\mathrm{JT}}^{i}-E_{\mathrm{band}}
$$

which is a measure of the electronic energy gain due to the inter-octahedral hopping, as opposed to the intraoctahedral JT energy $E_{\mathrm{JT}}$ (see Fig. 3). Note that a larger $\Delta E$ indicates a more favorable situation energetically. Since the lower JT-split level on each site is occupied in $\mathrm{LaMnO}_{3}$, the energy gain $\Delta E$ comes from hopping to the upper JT-split levels on the neighboring sites. Taking into account electron hopping to the four $\mathrm{NN}$ octahedra on the $\mathrm{Mn}-\mathrm{O}$ plane, one may estimate $\Delta E$ from the diagonalization of the $5 \times 5$ Hamiltonian matrix,

$$
H=\left(\begin{array}{ccccc}
-g Q & V & V & V & V \\
V & +g Q & 0 & 0 & 0 \\
V & 0 & +g Q & 0 & 0 \\
V & 0 & 0 & +g Q & 0 \\
V & 0 & 0 & 0 & +g Q
\end{array}\right),
$$

where $V$ is some measure of the inter-octahedral hopping strength and the expression Eq. (8) for the JT splitting has been used, with $G$ having been neglected for the sake

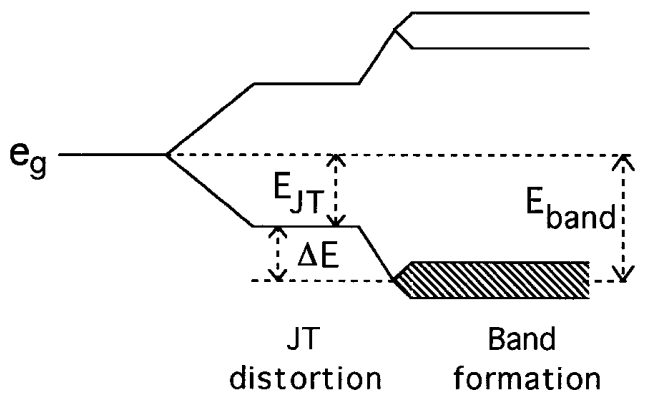

FIG. 3. Sketch of the JT splitting of the $e_{g}$ levels, which broaden into bands due to inter-octahedral electron hopping. Hatched area indicates the occupied bands.

of argument. Diagonalizing Eq. (11), one finds that the band energy is maximum at $Q=0$, varying monotonically between the two limits $\Delta E \rightarrow 2|V|-g Q$ as $Q \rightarrow 0$ and $\Delta E \rightarrow 4 V^{2} /(2 g Q)$ as $Q \rightarrow \infty$. The latter is simply the result of the second-order perturbation theory, which may be obtained by inspecting Eq. (11). It is clear that the band energy works against distortion.

The inter-octahedral hopping $V$ depends on the JT distortions of the neighboring sites, and to proceed further we must evaluate $\Delta E$ using Eqs. (6), (8), and (10) numerically. The results are presented in Fig. 4 for various distortions of the NN octahedra. We see that the configuration $\left(Q_{2},-Q_{2}\right)$ with alternating distortions of the $\mathrm{NN}$ octahedra is clearly preferred, the band energy gain being the highest there. Notice also the linear form of $\Delta E$ for small $Q$ as was argued above.

We have also calculated $\Delta E$ in the entire $\left(Q_{2}, Q_{3}\right)$ plane which is shown as a contour plot in Fig. 5(a) for the $\left(Q_{2}, Q_{3} ;-Q_{2}, Q_{3}\right)$-type staggered distortion of the NN octahedra [20]. The band energy is maximum at the center $Q=0$ and this is therefore what the band energy term would prefer. In that sense, the band term is similar to the elastic energy term $K Q^{2} / 2$, except that the band term has a strong directional dependence in the $\left(Q_{2}, Q_{3}\right)$ plane. Keeping in mind the proper limits for $\Delta E$ as discussed above, we fitted the band term in the entire $\left(Q_{2}, Q_{3}\right)$ plane with the following form: $\Delta E=$ const $+A /\left(Q+Q_{0}\right)-B Q^{2} \cos 3 \phi-C \cos 2 \phi$,

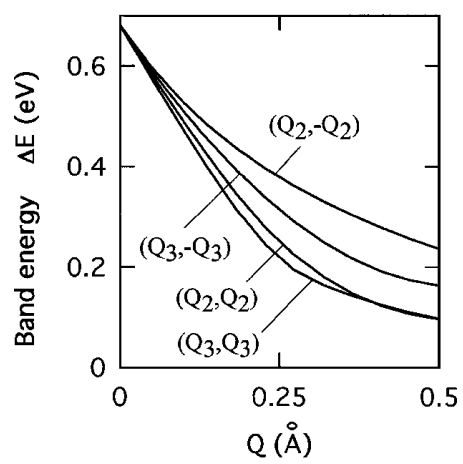

FIG. 4. Band energy $\Delta E$ as computed from Eq. (10). Here either the $Q_{2}$ or the $Q_{3}$ distortion is kept on the two JT centers in the unit cell as indicated in the figure. 


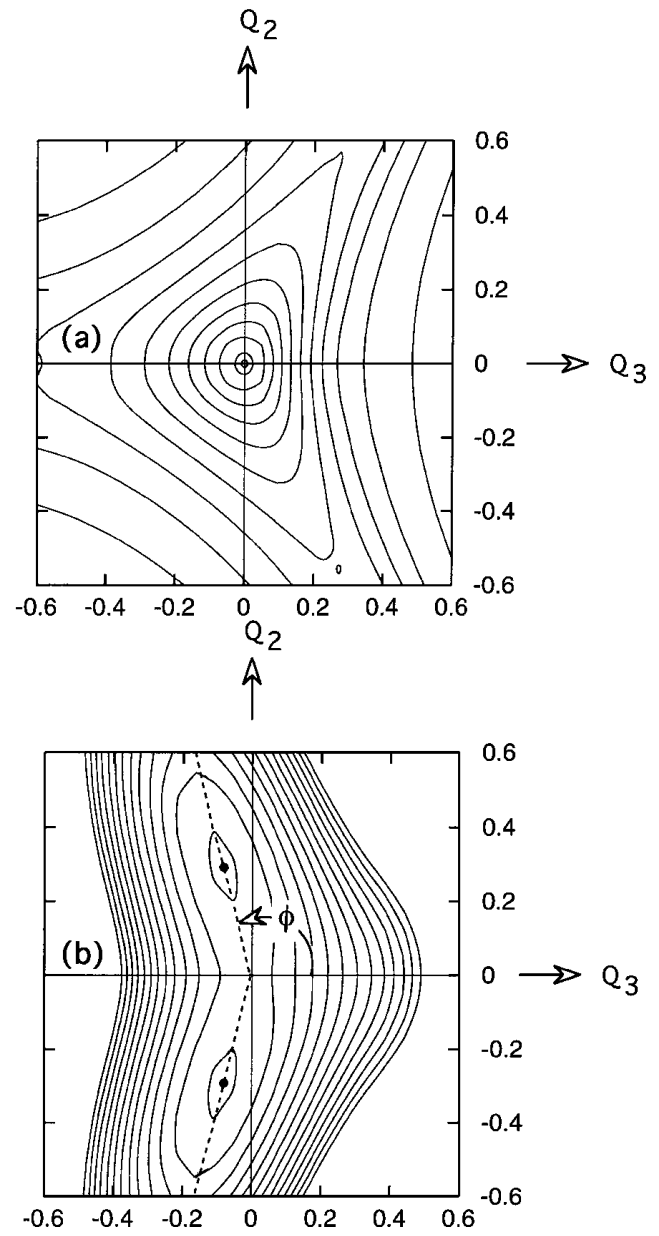

FIG. 5. (a) Energy contours of the band energy $\Delta E$ on the $\left(Q_{2}, Q_{3}\right)$ plane. $\Delta E$ is maximum at the center $(Q=0)$ and decreases differently along different directions such that the $Q_{3}$ direction is the least favored. (b) Same for the total energy obtained from Eq. (3), which shows two minima in the $Q_{2}-Q_{3}$ plane as opposed to three Mexican-hat-type minima for the isolated JT center. Parameters are $K=9 \mathrm{eV} / \AA^{2}, K^{\prime}=$ $1 \mathrm{eV} / \AA^{2}, g=2.0 \mathrm{eV} / \AA, G=1.5 \mathrm{eV} / \AA^{2}, V_{\sigma}=-0.52 \mathrm{eV}$, $V_{\sigma}^{\prime}=-0.42 \mathrm{eV}, V_{\pi}^{\prime}=0.09 \mathrm{eV}$, and $V_{\sigma}^{\prime \prime}=-0.06 \mathrm{eV}$.

with $A \approx 0.25 \mathrm{eV} \cdot \AA, B \approx 0.4 \mathrm{eV} / \AA^{2}, C \approx 0.03 \mathrm{eV}$, and $Q_{0} \approx 0.25 \AA$. As seen from this form, $\Delta E$ is reduced fastest along $Q_{3}(\phi=0)$, making the $Q_{3}$-type distortion relatively unfavorable.

For the isolated octahedron, the adiabatic potential surface has three minima along $\phi=0, \pm 2 \pi / 3$. Of these, the first direction is not favored by the band structure term in the crystal. The energy minima occur along the remaining two directions, although the band term changes the angle $\phi$ somewhat, as does the inter-octahedral elastic term $K^{\prime}$. The corresponding total energy contours are shown in Fig. 5(b). A small value of $K^{\prime} \approx 1 \mathrm{eV} / \AA^{2}$ was chosen for the contour plot, so that the minima occur at the point $(Q, \phi) \approx\left(0.3 \AA, 24^{\circ}\right)$, roughly the value observed in $\mathrm{LaMnO}_{3}$.
The electronic charge density corresponding to the two minima of the adiabatic potential surface consists of staggered $\left(z^{2}-1\right)$-type orbitals in the basal plane with the local $z$ axis along the long $\mathrm{Mn}-\mathrm{O}$ bond produced by the $Q_{2}$ distortion. This staggered ordering is long known both from experiments and from LDA calculations [21].

In conclusion, we have shown how the cooperative JahnTeller coupling between the individual $\mathrm{MnO}_{6}$ centers in the crystal leads to the simultaneous ordering of the octahedral distortion and the electronic orbitals. The basic ideas developed here should be applicable to a wide variety of crystals consisting of a lattice of interacting Jahn-Teller centers.

We thank the Research Board of the University of Missouri for partial financial support.

[1] A. J. Millis, Nature (London) 392, 147 (1998).

[2] G.-M. Zhao, K. Conder, H. Keller, and K. A. Müller, Nature (London) 381, 676 (1996); J. P. Franck, I. Isaac, W. Chen, J. Chrzanowski, and J.C. Irwin, Phys. Rev. B 58, 5189 (1998).

[3] S. Satpathy, J. Phys. Condens. Matter 10, L501 (1998); S. Satpathy, Solid State Commun. 112, 195 (1999).

[4] See, for example, I. B. Bersuker, The Jahn-Teller Effect and Vibronic Interactions in Modern Chemistry (Plenum Press, New York, 1984).

[5] J. H. Van Vleck, J. Chem. Phys. 7, 72 (1939).

[6] J. Kanamori, J. Appl. Phys. Suppl. 31, 14S (1960).

[7] K. I. Kugel and D. I. Khomskii, Sov. Phys. Usp. 25, 231 (1982).

[8] W. A. Harrison, Electronic Structure and the Properties of Solids (Freeman, San Francisco, 1979).

[9] C. Zener, Phys. Rev. 82, 403 (1951).

[10] P. W. Anderson and H. Hasegawa, Phys. Rev. 100, 675 (1955).

[11] S. Satpathy, Z. S. Popović, and F. R. Vukajlović, Phys. Rev. Lett. 76, 960 (1996); J. Appl. Phys. 79, 4555 (1996).

[12] W. E. Pickett and D. J. Singh, Phys. Rev. B 53, 1146 (1996).

[13] O. Jepsen and O. K. Andersen, Z. Phys. B 97, 35 (1995).

[14] M. Jaime, H. T. Hardner, M. B. Salamon, M. Rubinstein, P. Dorsey, and D. Emin, Phys. Rev. Lett. 78, 951 (1997).

[15] A. J. Millis, Phys. Rev. B 53, 8434 (1996).

[16] Y. Okimoto, T. Katsufuji, T. Ishikawa, A. Urushibara, T. Arima, and Y. Tokura, Phys. Rev. Lett. 75, 109 (1995).

[17] S. Y. Savrasov and D. Y. Savrasov, Phys. Rev. B 46, 12181 (1992).

[18] J. B. A. A. Elemans, B. van Laar, K. R. van der Veen, and B. O. Loopstra, J. Solid State Chem. 3, 238 (1971).

[19] H. Meskine and S. Satpathy, J. Appl. Phys. 85, 4348 (1999).

[20] We have also studied the distortions with different relative signs, viz., $\left( \pm Q_{2}, \pm Q_{3} ; \pm Q_{2}, \pm Q_{3}\right)$. The energy minima are the lowest for the $\left(Q_{2}, Q_{3} ;-Q_{2}, Q_{3}\right)$ case.

[21] See, e.g., Fig. 4 in Ref. [11], where the staggered ordering of the $\left(z^{2}-1\right)$ orbitals is implicit in the valence chargedensity contours calculated from LDA. 